\title{
Using Auditory and Visual Stimuli to Investigate the Behavioral and Neuronal Consequences of Reflexive Covert Orienting
}

\author{
Andrew H. Bell, Jillian H. Fecteau, and Douglas P. Munoz \\ Centre for Neuroscience Studies, Canadian Institutes of Health Research Group in Sensory-Motor Systems, Department of Physiology, \\ Queen's University, Kingston, Ontario K7L 3N6, Canada
}

Submitted 6 November 2003; accepted in final form 17 December 2003

\begin{abstract}
Bell, Andrew H., Jillian H. Fecteau, and Douglas P. Munoz. Using auditory and visual stimuli to investigate the behavioral and neuronal consequences of reflexive covert orienting. J Neurophysiol 91: 2172-2184, 2004. First published December 31, 2003; 10.1152/jn.01080.2003. Reflexively orienting toward a peripheral cue can influence subsequent responses to a target, depending on when and where the cue and target appear relative to each other. At short delays between the cue and target [cue-target onset asynchrony (CTOA)], subjects are faster to respond when they appear at the same location, an effect referred to as reflexive attentional capture. At longer CTOAs, subjects are slower to respond when the two appear at the same location, an effect referred to as inhibition of return (IOR). Recent evidence suggests that these phenomena originate from sensory interactions between the cue- and target-related responses. The capture of attention originates from a strong target-related response, derived from the overlap of the cueand target-related activities, whereas IOR corresponds to a weaker target-aligned response. If such interactions are responsible, then modifying their nature should impact the neuronal and behavioral outcome. Monkeys performed a cue-target saccade task featuring visual and auditory cues while neural activity was recorded from the superior colliculus (SC). Compared with visual stimuli, auditory responses are weaker and occur earlier, thereby decreasing the likelihood of interactions between these signals. Similar to previous studies, visual stimuli evoked reflexive attentional capture at a short CTOA (60 ms) and IOR at longer CTOAs (160 and $610 \mathrm{~ms}$ ) with corresponding changes in the target-aligned activity in the SC. Auditory cues used in this study failed to elicit either a behavioral effect or modification of SC activity at any CTOA, supporting the hypothesis that reflexive orienting is mediated by sensory interactions between the cue and target stimuli.
\end{abstract}

\section{IN T R O D U C T I O N}

A sudden change in our environment, such as a mouse scurrying across the floor, reflexively grabs our attention. The consequences of this reflexive capture of attention can be investigated using the cue-target saccade task (Posner and Cohen 1984). In this task, a brief flash of light or burst of noise in the periphery is used to draw the subject's attention to its location. After a variable delay, a target appears at the same or opposite location as the cue with equal probability to which the subject must generate a response. Measuring the time required by the subject to generate a response to the target can assess the behavioral consequences of the initial reflexive shift in attention (Jonides 1981; Maylor and Hockey 1985; Posner 1978; Posner and Cohen 1984).

The location of the cue relative to the target and the time that

Address for reprint requests and other correspondence: D. Munoz, Department of Physiology, Queen's University, Kingston, Ontario K7L 3N6, Canada (E-mail: doug@eyeml.queensu.ca). elapses between their respective onsets [defined as the cuetarget onset asynchrony (CTOA)] both influence the subject's response time. At short CTOAs [i.e., $<200 \mathrm{~ms}$ for humans, $<80 \mathrm{~ms}$ for monkeys (Fecteau and Munoz 2003)], subjects respond faster when the cue and target appear at the same location compared with when they appear at opposite locations. This same-location advantage is thought to represent the "reflexive capture of attention" (Jonides 1981; Posner and Cohen 1984; Remington et al. 1992; see Egeth and Yantis 1997 for review). At longer CTOAs [i.e., >200 ms for humans (Fecteau and Munoz 2003); $>80 \mathrm{~ms}$ for monkeys (Dorris et al. 2002; Fecteau and Munoz 2003)], subjects respond more slowly when the cue and target appear at the same location. This has been labeled "inhibition of return" (IOR; Posner et al. 1985; see also Maylor and Hockey 1985; Posner and Cohen 1984) and it is thought to represent the adaptive bias of observers to explore novel locations in the environment.

Recent studies have demonstrated a neurophysiological correlate to these consequences of reflexive orienting. Dorris and colleagues (2002) demonstrated that increased saccadic reaction times (SRTs) to previously cued locations were associated with a decrease in the magnitude of the neural response to the target in the superior colliculus (SC). Fecteau and Munoz (2003) expanded on these results by demonstrating that reflexive attentional capture was associated with an increase in the magnitude of the target-aligned response. Importantly, these two studies further demonstrated that these changes in activity were limited to the target-aligned response and were not observed in the motor burst accompanying the saccade. These data suggest that the behavioral consequences of covert orienting, in this case the reflexive capture of attention and IOR, may be linked to bottom-up, sensory processes. The reflexive capture of attention originates from the overlap of the cue- and target-aligned responses at short CTOAs, whereas IOR originates from a reduced target-aligned response at longer CTOAs.

If such sensory interactions between the cue- and targetaligned responses are involved in producing the reflexive capture of attention and IOR, then modifying these interactions should directly influence the neuronal and behavioral outcomes. We assessed this possibility by training monkeys to perform a cue-target saccade task in which both auditory and visual cues preceded visual targets. While the animals performed this task, we recorded single-unit activity from neurons in the intermediate layers of the SC. Compared with visual stimuli, initial phasic responses to auditory stimuli in the SC

\footnotetext{
The costs of publication of this article were defrayed in part by the payment of page charges. The article must therefore be hereby marked "advertisement" in accordance with 18 U.S.C. Section 1734 solely to indicate this fact.
} 
occur much earlier, are weaker, and are shorter in duration (Bell et al. 2001; Wallace et al. 1996). Accordingly, we predict that these properties of auditory responses will provide less opportunity for the auditory cue-aligned activity and visual target-aligned activity to interact and the reflexive capture of attention will not be observed in behavior at short CTOAs. Likewise, if the behavioral expression of IOR depends on an attenuated sensory response to the target that occurs as a result of the same neurons responding to both the visual cue and target early in sensory processing, then no such attenuation should occur after auditory cues and IOR should not be seen. Moreover, if the differential response properties for visual and auditory stimuli are responsible for the inability of auditory stimuli to induce reflexive attentional capture or IOR, this may explain why crossmodal IOR is difficult to observe in behavior (see Reuter-Lorenz and Rosenquist 1996; Spence et al. 1998), even though it is demonstrated easily within individual modalities (e.g., Poliakoff et al. 2002; Spence et al. 2000).

Preliminary data were previously presented in abstract form (Bell and Munoz 2002).

\section{METHODS}

\section{Preparation of experimental animals}

All procedures were approved by the Queen's University Animal Care Committee and were in accordance with the Canadian Council on Animal Care policy for the use of laboratory animals. Two adult male rhesus monkeys (Macaca mulatta), weighing about 7 and $10 \mathrm{~kg}$, were used in this study. Animals were prepared for chronic experiments in one aseptic surgical session (see Munoz and Istvan 1998 for details). Scleral search coils to monitor eye movements and a headrestraint device were implanted. A stainless steel recording chamber, centered on the midline and tilted $38-40^{\circ}$ posterior of vertical, was implanted to allow recordings from both the right and left SC. Animals were given a course of antibiotic and analgesic treatment and monitored closely after surgery. They were allowed a recovery period of at least $2 \mathrm{wk}$ before behavioral training was initiated.

\section{Behavioral paradigm}

The monkeys were trained to perform a nonpredictive cue-target saccade task including auditory and visual cues combined with visual targets (Fig. 1). The onset of each trial was signaled by the removal of a background light and presentation of a laser- or light-emitting diode (LED)-generated central fixation point (FP) back-projected onto a tangent screen facing the animal. The animal was required to fixate the FP for a period of $600-800 \mathrm{~ms}$, after which an auditory or visual cue was presented in the periphery for $50 \mathrm{~ms}$. The cue was extinguished and the animal was required to maintain central fixation for an additional 10, 110, or $560 \mathrm{~ms}$ (corresponding to CTOAs of 60,160 , and $610 \mathrm{~ms}$ ). The FP was extinguished and a visual target appeared at the SAME location as the peripheral cue or at the diametrically OPPOSITE location, with equal probability. The three CTOAs were selected on the basis of a pilot behavioral study using a wide range of CTOAs (Dale MK, AH Bell, and DP Munoz, unpublished observations), to obtain behavioral correlates of reflexive attentional capture and IOR.

The visual cue and target stimuli were generated using LEDs (0.05 $\mathrm{cd} / \mathrm{m}^{2}$ ). The auditory-cue stimulus was composed of a white noise burst ( $43.5 \mathrm{~dB}, \mathrm{~A}$-scale, measured from the monkey's head), produced by small $4-\mathrm{cm}, 8.0-\Omega$ speakers suspended in front of the tangent screen, facing the animal. The speakers were positioned immediately adjacent to where the visual stimuli would appear so as not to obstruct them. The intensities of the auditory and visual stimuli were selected

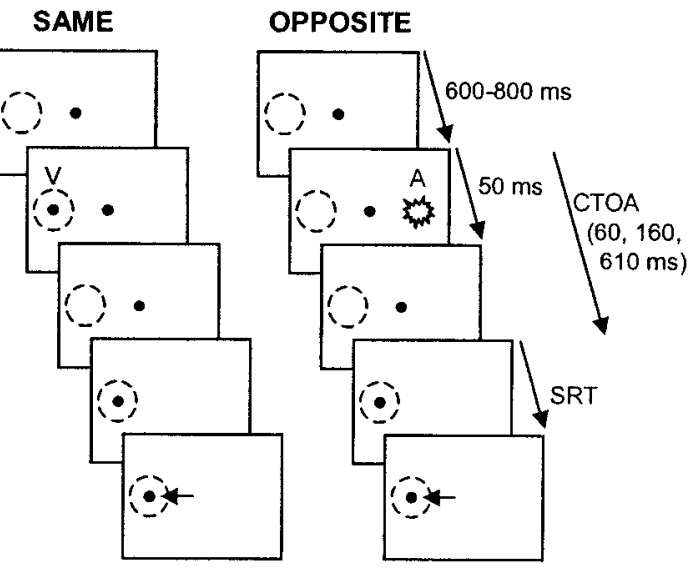

FIG. 1. Schematic representation of the nonpredictive cue-target saccade task (see METHODS for details). Monkeys are required to maintain stable gaze on a central fixation point while a visual (V) or auditory (A) cue is presented into the receptive field of the neuron (indicated by the dashed circle) or to the opposite location. After which, the cue is extinguished and the animals must maintain fixation for an additional period until the fixation point is removed and the visual target is presented, either to the same location as the cue or to the diametrically opposite location, with equal probability. Monkeys are then required to generate a saccade to the target. Temporal delay between cue and target onset is defined as the cue-target onset asynchrony (CTOA). SRT, saccadic reaction time.

because they are known to evoke spatially dependent multisensory interactions (Bell et al. 2001, 2002) and the strength of these interactions are known to be strongest for pairings of weaker stimuli (see Stein and Meredith 1993). All trial types (auditory vs. visual cue; SAME vs. OPPOSITE; 60-, 160-, and 610-ms CTOA) were randomly interleaved within a single block of trials. Monkeys were given a liquid reward if they maintained central fixation (i.e., kept their eyes stable within $2^{\circ}$ of the FP) for the duration of the fixation period and generated a saccade to the visual target. They worked until fully satiated, at which point they were returned to their home cages. Daily records of animal weight and water intake were kept and the animals' health was closely monitored by the institute veterinarian.

\section{Recording techniques and receptive field mapping}

Single-neuron activity was recorded extracellularly in both SC with tungsten microelectrodes (Frederick Haer) having impedances of $0.5-3 \mathrm{M} \Omega$ at $1 \mathrm{kHz}$. Electrodes were lowered by a hydraulic microdrive (Narishige) through stainless steel guide tubes supported by a Delrin grid placed inside the recording chamber (Crist et al. 1988). Single-neuron activity was sampled at $1 \mathrm{kHz}$ after passing through a window discriminator (Bak Electronics), which excluded action potentials that did not meet both amplitude and temporal constraints. Control of the behavioral paradigms as well as storage of eye position and neural data was controlled by Pentium PC running a real time data acquisition software package (REX Ver. 5.4; Hays et al. 1982). Eye position was sampled at $500 \mathrm{~Hz}$.

To map the extent of a neuron's visual receptive field, a handheld ophthalmoscope was used to back-project moving spots and bars of light onto the tangent screen while the monkey maintained central fixation. In addition, visual stimuli were systematically presented throughout the visual field. The center of the receptive field was approximated as the point where the maximum visual response was elicited. Because previous studies have shown that auditory receptive fields in the primate SC tend to be very large $\left(45-180^{\circ}\right.$ in the contralateral hemifield in primates; Wallace et al. 1996), no attempt was made to map the extent of the auditory receptive fields and the auditory stimuli were placed immediately adjacent to the visual stimuli for each of the cue positions. 


\section{Data analysis}

All data analysis was carried out on a Sun Ultra 60 Sparcstation using user-generated programs and a Pentium PC running MatLab software (Mathworks). Data were first run through an automated saccade-detection program, which identified the beginning and end of each saccade based on velocity and acceleration template matching (Waitzman et al. 1991). All marks were later verified by the experimenter and adjusted if necessary. Before analysis, all incorrect trials were rejected (monkey generated saccade to cued location in OPPOSITE trials, i.e., "direction error"; see following text; or generated saccade before the removal of the FP). Likewise, saccades with latencies below $70 \mathrm{~ms}$ or above $500 \mathrm{~ms}$ were excluded.

Neuronal responses were analyzed by constructing spike density functions based on an exponential growth/decay function. The spike density waveform was obtained by convolving each spike with the following function (Thompson et al. 1996)

$$
A(t)=1-\exp \left(\frac{-t}{\tau_{g}}\right) \exp \left(\frac{-t}{\tau_{d}}\right)
$$

where the activation level (A) varies as a function of time $(\mathrm{t})$, according to $\tau_{\mathrm{g}}$, the growth time constant that was set to $1 \mathrm{~ms}$, and $\tau_{\mathrm{d}}$, the decay time constant that was set at $20 \mathrm{~ms}$. The individual pulses were then summed to generate a single spike density function for each trial. To determine a neuron's responsiveness to the different stimulus modalities, the peak response from 0 to $150 \mathrm{~ms}$ after cue presentation at the $610-\mathrm{ms}$ CTOA trials was measured. A given neuron was classified as a visual and/or auditory-responsive neuron when the magnitude of its cue-aligned activity was significantly greater than baseline (defined as mean activity 100-0 ms before cue onset; Wilcoxon rank-sum test, $P<0.05$ ). To determine a neuron's saccadealigned response, the peak activity $\pm 10 \mathrm{~ms}$ surrounding saccade onset was measured. A neuron was defined as saccade-related when the peak saccade-aligned activity consistently exceeded $80 \mathrm{spikes} / \mathrm{s}$ for saccades to the neuron's preferred direction. The experimenter later verified all classifications to ensure accuracy and consistency.

The cue-response onset latency was defined as the point where the activation level exceeded baseline (defined as the mean activity 400$200 \mathrm{~ms}$ before cue onset) plus 3 SDs. The activity had to remain above this level for a minimum of $10 \mathrm{~ms}$ to be classified as a valid response. To quantify the magnitude of activity after target presentation (i.e., the "peak of the target-aligned burst"), the absolute peak spike density 50-150 ms after target presentation was measured. To estimate the magnitude of the "target-related response" (i.e., the magnitude of the sensory response to the target, independent of differences in baseline activity produced by the cue; see RESULTS), the pretarget activity (mean activity 35-40 ms after target presentation) was subtracted from the target-aligned burst of activity.

For the population analysis of each dependent variable, repeatedmeasures ANOVAs, including the variables: cue modality (visual vs. auditory), cue condition (SAME vs. OPPOSITE location), and CTOA (60, $160,610 \mathrm{~ms}$ ) were used. Simple effects were analyzed with pairwise Wilcoxon signed rank-sum tests. Individual sessions were analyzed with Wilcoxon rank-sum tests. In all instances, an alpha of 0.05 was chosen as significant. For display purposes only, spike density functions are shown as floating averages of 10 -ms bin widths.

Only the data from correct trials were included in the analyses described below. Error trials were divided into one of two types: anticipatory responses, where the saccade was generated to the correct location but with an SRT $<70 \mathrm{~ms}$; and direction errors, defined as saccades initially generated away from the target (e.g., to the cued location in the OPPOSITE condition). Monkeys generated a total of 1,072 anticipation errors (8\%), with the majority being generated in response to the visual cue and at the 160 -ms CTOA [cue modality: $F(1,55)=9.885, P<0.005$; cue condition: $F(1,55)=0.207, P>$ 0.50 ; CTOA: $F(2,54)=5.333, P<0.01]$. Monkeys generated a total of 520 direction errors (4\%), with the majority being generated in response to the visual cue at a CTOA of $610 \mathrm{~ms}$ and in the OPPOSITE condition [cue modality: $F(1,55)=26.464, P<0.001$; cue condition: $F(1,55)=13.308, P<0.001$; CTOA: $F(2,54)=15.763, P<0.001]$.

\section{RES ULTS}

\section{Behavior}

Two monkeys completed a total of 12,305 correct trials over the course of the recording sessions. Because both monkeys exhibited similar behavior, data have been grouped across the two subjects. Cue modality (visual vs. auditory), cue condition (SAME vs. OPPOSITE), and CTOA (60, 160, $610 \mathrm{~ms}$ ) interacted with one another to influence SRT [3-way interaction: $F(2,112)=20.059, P<0.0001]$. To facilitate the presentation of these data, results for each cue modality are described separately.

On visual-cue trials (Fig. $2 A$; black lines) at the shortest CTOA (60 ms), monkeys generated saccades with significantly shorter SRTs when the cue and target appeared at the SAME
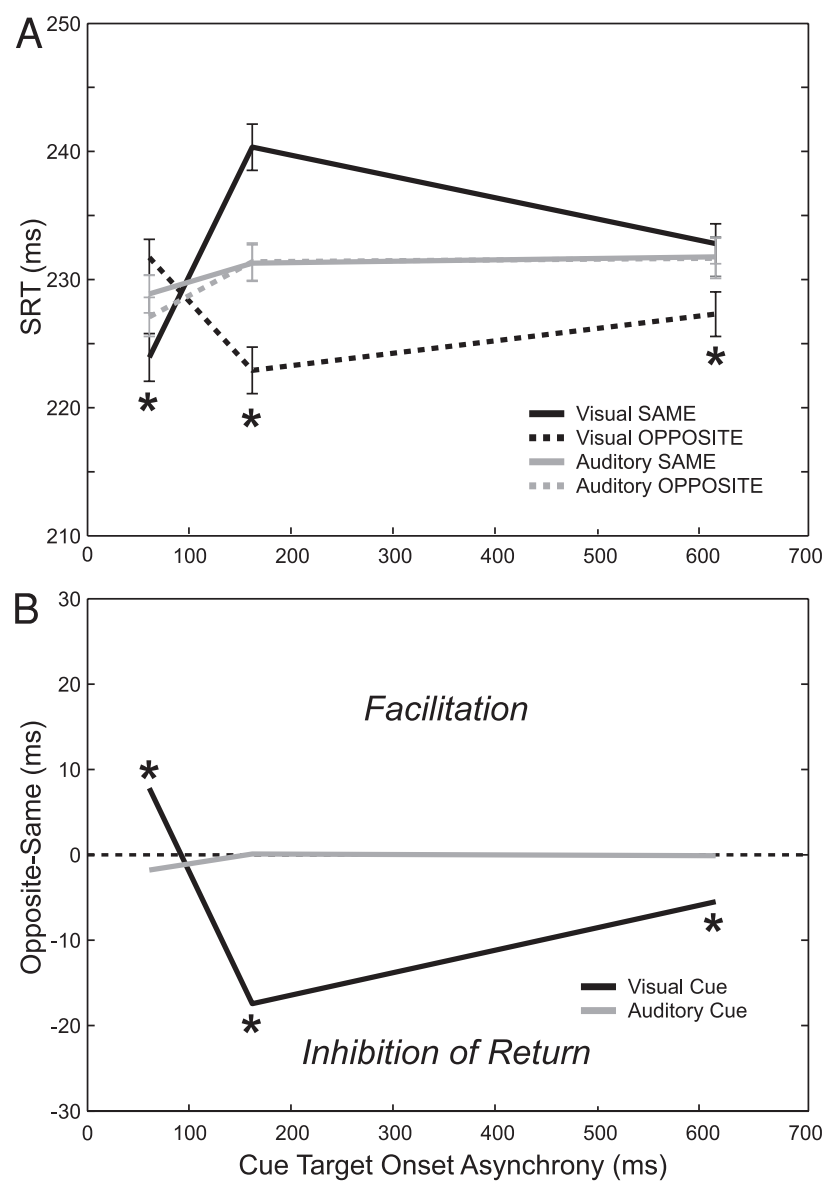

FIG. 2. Behavior in the nonpredictive cue-target saccade task. A: mean (and $\mathrm{SE}$ ) saccadic reaction times for the two monkeys. Visual-cue trials shown in black; auditory-cue trials shown in gray. Solid lines indicate trials where the cue and target appeared in the SAME location; dashed lines represent trials where the cue and target appeared in OPPOSITE locations. $B$ : mean SRT for the SAME condition subtracted from the mean SRT for the OPPOSITE condition. Values $>0$ indicate shorter SRTs in the SAME condition (i.e., facilitated responses); values $<0$ indicate shorter SRTs in the OPPOSITE condition (i.e., inhibition of return). Asterisks indicate statistically significant differences between the SAME and OPPOSITE conditions for the given cue modality (Wilcoxon rank-sum test, $P<0.05$ ). 
location (solid black lines) compared with when the stimuli appeared at OPPOSITE locations (dashed black lines; Wilcoxon rank-sum test, $P<0.0001$ ). At the intermediate CTOA (160 $\mathrm{ms}$ ), this effect was reversed and mean SRTs in the SAME condition were significantly longer compared with the OPPOSITE condition $(P<0.0001)$. Note this difference was attributed both to a significant increase in mean SRT for the SAME condition $(P<0.0001)$ and a significant decrease in mean SRT for the OPPOSITE condition $(P<0.0001)$, compared with the corresponding trial type at the 60-ms CTOA. At the longest CTOA tested $(610 \mathrm{~ms})$, a significant albeit reduced oppositelocation advantage was present $(P<0.0001)$. Subtracting the mean SRT in the SAME condition from that of the OPPOSITE condition for each CTOA (Fig. $2 B$, black line) revealed a behavioral facilitation, defined here as reflexive attentional capture, at a CTOA of $60 \mathrm{~ms}$ and IOR at CTOAs of 160 and $610 \mathrm{~ms}$.

By contrast, when an auditory cue preceded the visual target (Fig. 2; gray lines), no differences between the SAME and OPPOSITE conditions were obtained at any of the CTOAs tested (Wilcoxon rank-sum test, $P$ values $>0.50$ ). Saccades generated to visual targets after auditory cues had very similar SRTs, regardless of the location of the cue relative to the target or the CTOA. Interestingly, mean SRT for auditory-cue trials consistently fell between those obtained for the two visual-cue conditions. That is, at the $60-\mathrm{ms}$ CTOA, presenting an auditory cue before a visual target did not provide as great a behavioral advantage as a visual cue and target presented to the SAME position but, rather, elicited shorter SRTs compared with a visual cue and target presented to OPPOSITE locations. At the longer CTOAs (160 and $610 \mathrm{~ms}$ ), auditory cues had little effect on behavior-effectively serving as a neutral condition, and therefore emphasizing how the IOR effect obtained after visual cues was likely attributable to both an inhibitory influence acting in the SAME condition and a facilitatory effect acting in the OPPOSITE condition.

We also assessed the effect of cue modality, cue condition, and CTOA on the execution of saccades by comparing peak saccadic velocity and the accuracy of the saccadic endpoints across the different conditions. Main sequence plots (saccadic amplitude vs. peak saccadic velocity; Bahill et al. 1975) were constructed for each trial condition (not shown). Peak saccadic velocities were not influenced by cue modality, cue condition, or CTOA [3-way: $F(2,112)=1.845, P=0.158$ ]. For a given saccadic amplitude, a similar peak saccadic velocity was achieved, regardless of the individual trial conditions.

To assess the accuracy of the saccades, we calculated both the saccadic gain (saccadic amplitude/target eccentricity) and endpoint error (magnitude of the vector between saccadic endpoint and true target location; not shown). Neither of these factors was influenced by cue condition, modality, or CTOA [3-way: Gain: $F(2,112)=0.641, P=0.527$; Motor Error: $F(2,112)=1.773, P=0.170]$. All saccades were equally accurate, regardless of the modality of the cue, the location of the target relative to the cue, or the delay between cue and target onset.

In summary, reflexively shifting attention to a particular location by means of a visual cue resulted in early reflexive attentional capture at the short CTOA $(60 \mathrm{~ms})$ followed by IOR at the longer CTOAs (160 and $610 \mathrm{~ms})$. These effects were limited to changes in SRT; peak saccadic velocity and endpoint accuracy were unaffected. Auditory cues were unable to elicit either reflexive attentional capture or IOR at any CTOA tested, showing no significant change in SRT across any of the trial types.

\section{Activity in the SC recorded from the nonpredictive cue-target saccade task}

Sixty-one neurons were recorded from the intermediate layers of both colliculi of two monkeys ( 29 and 32 from monkeys $\mathrm{R}$ and $\mathrm{H}$, respectively). Eighty-four percent $(51 / 61)$ were responsive to sensory stimuli (visual only: $n=37$; auditory only: $n=2$; bimodal: $n=12$ ) and were considered for further analysis. Of these, $86 \%$ (44/51) had significant saccade-related activity (see METHODS for classification criteria).

The behavioral consequences of nonpredictive cueing were closely reflected in changes in the magnitude of the targetaligned burst of activity of neurons in the intermediate layers of the SC [3-way interaction between cue modality, cue condition, and CTOA: $F(2,96)=11.099, P<0.001]$. An analysis of the magnitude of the saccadic burst showed no difference across the different trial conditions [main effect of cue condition: $F(1,48)=0.008, P>0.50$; cue modality: $F(1,48)=$ $0.965, P>0.32$; CTOA: $F(2,96)=0.783, P>0.45$; or 3-way: $F(2,96)=1.649, P>0.19]$. When considered in conjunction with the lack of effect of cue modality, condition, or CTOA on the peak velocity and saccadic endpoint accuracy, these data suggest that reflexively orienting by means of a nonpredictive cue influences the neural processes involved in saccadic initiation but not those associated with the actual execution of the saccade. We now describe how the early and late consequences of reflexive covert orienting (i.e., attentional capture and IOR) were correlated to the peak target-aligned burst of activity in the SC.

\section{Neural correlates of reflexive attentional capture}

At the shortest CTOA tested (60 ms), monkeys exhibited a same-location advantage when the target was preceded by a visual cue and no difference in behavior when an auditory cue was presented (Fig. 2). Does the presence of the same-location advantage for visual cues correspond to an overlap of the cueand target-aligned responses that does not occur after auditory cues?

Figure 3 shows the activity of a single sensory-motor neuron in the intermediate layers of the SC when visual or auditorycue stimuli were presented at a 60-ms CTOA. When the visual cue appeared in the receptive field of this neuron (Fig. 3, $A$ and $E$ ), a strong burst of action potentials occurred, beginning about $65 \mathrm{~ms}$ after cue onset. When the visual target appeared at the SAME location as the visual cue (Fig. $3 A$ ), the neural response to the target added to the residual activity from the cue, resulting in a stronger target-aligned burst compared with when the cue was presented on the opposite side (Fig. 3C).

This overlap between the cue- and target-aligned responses did not occur after auditory cues (Fig. 3, $B, D, F$, and $H$ ). This particular neuron had a weak, but significant, response to the auditory cue that was long over by the time the visual response to the target began. As such, there was no opportunity for the cue- and target-aligned responses to overlap when both stimuli appeared in the same receptive field (Fig. 3B). Moreover, there 


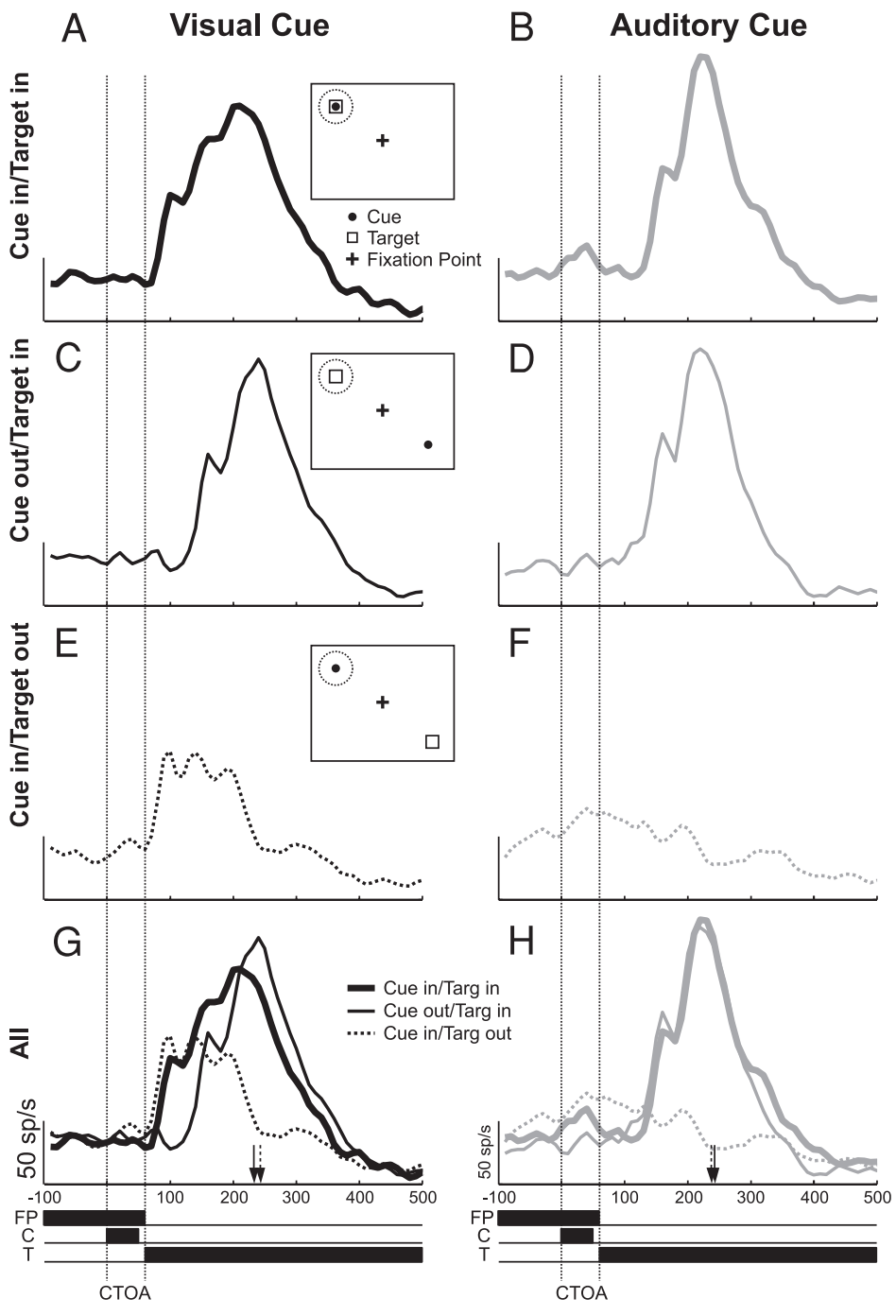

Time from Cue Onset (ms)
FIG. 3. Neuronal correlates of reflexive attentional capture in the superior colliculus (SC). Activity for an individual sensory-motor neuron recorded from the intermediate layers of the SC, for trials at the $60-\mathrm{ms}$ CTOA. All traces are aligned on cue onset. When a visual cue and target appear in the same response field ( $A$, black line), there is a substantial increase in the magnitude of the target-aligned activity compared with when the cue appears at the OPPOSITE location, outside the receptive field of the neuron $(C)$. The auditory-cue response, on the other hand, provided very little residual cue activity on which the visual target response could build and so no facilitation occurred $(B)$. Vertical arrows indicate the mean SRT for the SAME (solid arrow) and OPPOSITE (dashed arrow) conditions. was no other observable change in the target-aligned response after the appearance of the auditory cue. Therefore it seems unlikely that any significant multisensory interactions (Stein and Meredith 1993) took place after presentation of the auditory cue and visual target at the 60-ms CTOA.

Similar effects of cue modality and cue condition were seen across the population of neurons examined. Figure 4 shows the mean peak of the target-aligned burst for each visually responsive neuron ( $n=49$; Fig. 4). Note that for the remainder of the analysis, the auditory-only neurons $(n=2)$ have been removed because they had no target-related activity. As demonstrated in Fig. 3, the overlap of the visual-cue and target-aligned re-

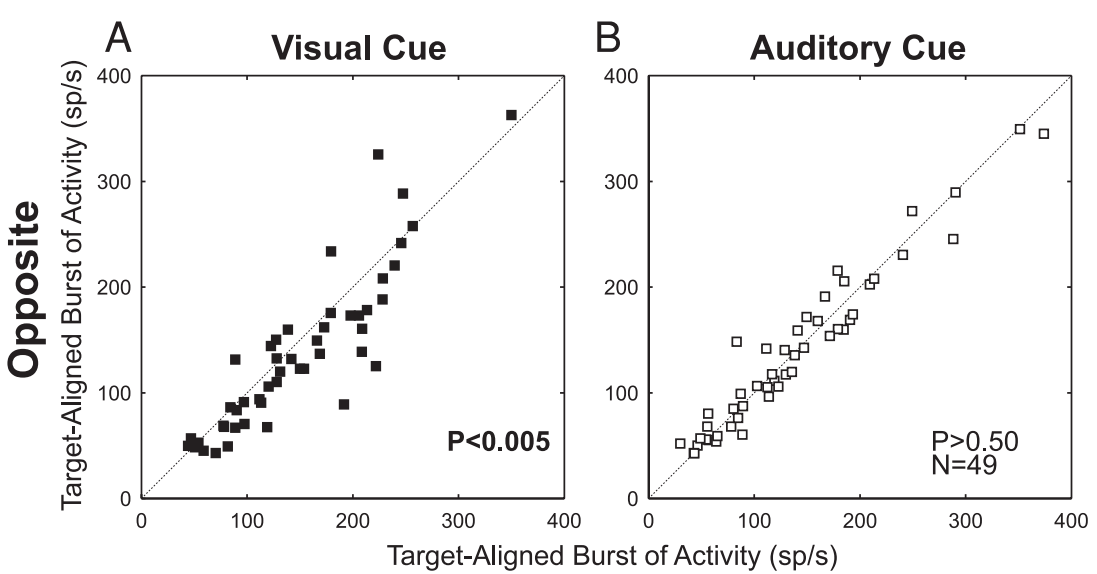

FIG. 4. Influence of cue condition and modality on neuronal activity at the 60-ms CTOA. Each point represents the mean peak in the target-aligned burst of activity for an individual visually responsive neuron, after a visual $(A)$ or auditory $(B)$ cue. Points lying below the unity line indicate greater activity in the SAME condition and vice versa. Across the population, there was a highly significant trend for a stronger burst of target-aligned activity in the SAME condition compared with the OPPOSITE condition after visual $(A$, solid points, Wilcoxon signed rank-sum test; $P<0.005$ ) but not auditory cues $(B$, open points; $P>0.50)$.

\section{Same}


sponses at the 60 -ms CTOA resulted in a significantly stronger burst of activity in the SAME condition compared with the OPPOSITE condition for the majority $(36 / 49 ; 73 \%)$ of the neurons examined (Fig. 4A; $P<0.005$ ). This was associated with a same-location advantage in behavior (Fig. 2).

No change in the target-aligned burst was observed after the presentation of auditory cues (Fig. 4B). Relatively few neurons in our sample responded to auditory stimuli $(14 / 61 ; 23 \%)$ and those that did exhibited weaker responses (Wilcoxon rank-sum test; $P=0.05)$ that occurred earlier in time compared with visual responses (Wilcoxon rank-sum test; $P<0.0001$; Table 1). As such, there was no opportunity for the auditory cue- and visual target-aligned responses to interact. In the auditory-cue condition, the magnitude of the target-aligned burst of activity did not change on the basis on the cue's location (i.e., SAME vs. opposite; Fig. $4 B ; P>0.50$ ).

To summarize, the same-location advantage that was obtained in SRT for visual cues at the 60-ms CTOA was associated with an increase in the peak of the target-aligned burst of activity that originated from the overlap of the cue- and targetaligned responses. The inability of auditory stimuli to evoke a similar pattern of behavior appears to be a result of both a lack of significant cue-aligned response across the population on which the incoming target signals can build and the absence of significant multisensory interactions between the cue and target responses.

\section{Neural correlates of inhibition of return}

At the 160- and 610-ms CTOAs, an opposite-location advantage in behavior emerged for visual but not auditory cues (Fig. 2). Figure 5 shows the activity of a sensory-motor neuron recorded from the intermediate layers of the $\mathrm{SC}$ at the 160- and 610-ms CTOAs. In the case of visual-cue trials, the opposite-location advantage in behavior was associated with a reduction in the magnitude of the target-aligned burst of activity in the SAME condition compared with when the two were presented to OPPOSITE locations. This reduction of target-aligned activity was greater at the $160-\mathrm{ms}$ CTOA (Fig. 5A) compared with the 610-ms CTOA (Fig. 5C), as was the behavioral IOR effect (Fig. 2). For auditory cues, neither the relative location of the cue nor the CTOA had an effect on behavior (Fig. 2) or the target-aligned burst of activity (Fig. 5, $B$ and $D$ ).

Similar trends were observed across the population of neurons sampled (Fig. 6). At the 160-ms CTOA, visual-cue trials consistently exhibited weaker target-aligned bursts of activity

TABLE 1. Properties of the cue-related response

\begin{tabular}{ccr}
\hline \hline \multicolumn{1}{c}{ Cues } & $\begin{array}{c}\text { Cue Response Onset } \\
\text { Latency, ms }\end{array}$ & $\begin{array}{c}\text { Cue Response } \\
\text { Magnitude, sp/s }\end{array}$ \\
\hline Visual & $75 \pm 2(52-118)$ & $1301 \pm 10(43-342)$ \\
$60 \mathrm{~ms} \mathrm{CTOA}$ & $76 \pm 2(52-121)$ & $132 \pm 10(43-335)$ \\
$160 \mathrm{~ms}$ CTOA & $75 \pm 2(53-118)$ & $133 \pm 10(41-369)$ \\
$610 \mathrm{~ms}$ CTOA & $74 \pm 2(49-115)$ & $129 \pm 9(43-325)$ \\
Auditory & $44 \pm 3(30-67)$ & $94 \pm 12(47-189)$ \\
$60 \mathrm{~ms}$ CTOA & $44 \pm 3(30-67)$ & $95 \pm 13(43-204)$ \\
$160 \mathrm{~ms}$ CTOA & $44 \pm 3(30-68)$ & $96 \pm 12(53-191)$ \\
$610 \mathrm{~ms}$ CTOA & $44 \pm 3(31-67)$ & $90 \pm 12(45-173)$ \\
\hline
\end{tabular}

Values are mean \pm SE. For visually responsive neurons $(n=49)$; for all aurally responsive neurons $(n=14)$. CTOA, cue-target onset asynchrony.
(Fig. 6A; Wilcoxon signed-rank test, $P<0.0001$ ) in the SAME condition. At the longest CTOA (610 ms), although a significant opposite-location advantage persisted in behavior, the difference in activity between the SAME and OPPOSITE conditions with visual cues was no longer significant (Fig. $6 C ; P>0.20$ ). It is possible that an increase in pretarget activity observed after the cue response might have masked a difference in the actual sensory response to the target. This possibility is explored further in the next section. Again, no difference in activity was observed after auditory cues at either CTOA (Fig. $6, B$ and $D$ ).

To illustrate these data more clearly, we plotted the neural activity in a similar manner as SRT (compare Figs. 7 and 2). This subtraction plot shows that a greater peak target-aligned burst of activity in the SAME condition was associated with a same-location advantage in behavior, whereas weaker activity was associated with an opposite-location advantage (compare Figs. $7 B$ and $2 B$ ). As predicted on the basis of behavior, the opposite-location advantage in mean SRT at the 160-ms CTOA for visual-cue trials corresponded to both a significant reduction in the target-aligned burst in the SAME condition and a significant increase in the OPPOSITE condition, relative to the visual-cue trials at the 60 -ms CTOA (Wilcoxon signed-rank test, $P$ values $<0.001$ and 0.001 , respectively).

To confirm the existence of a relationship between SRT and the magnitude of the target-aligned burst of activity, we calculated the Pearson correlation coefficient for SRT versus the peak of the target-aligned burst of activity on a trial-by-trial basis for each neuron in our sample population. For this analysis, we grouped trials from all CTOAs and both cue modalities and conditions. Figure $8 A$ shows the correlation between SRT and the peak of the target-aligned burst of activity on a trial-by-trial basis for a single sensory-motor neuron (black points, Fig. 8A). This particular neuron showed a significant negative relationship such that as the magnitude of the peak activity increased, SRT decreased (Pearson correlation coefficient: $r=-0.42 ; P<0.05)$. This was also true for the population (Fig. $8 B$ ), which exhibited a negative bias in correlation coefficients and of which over half of the neurons assessed $(32 / 49 ; 65 \%)$ were significantly correlated $(P<0.05)$.

These data demonstrate that sensory interactions between the cue and target influence the magnitude of the target-aligned burst of neurons in the intermediate layers of the SC and how these changes are related to behavior. Reflexive attentional capture was associated with an increase in activity, arising from the overlap of the cue- and target-related responses. IOR was associated with a reduction in the peak of the target-aligned burst of activity in the SAME condition and an increase in the OPPOSITE condition. Auditory cues produced neither of these neural trends, which could account for why no crossmodal reflexive attentional capture or IOR was observed in our task.

\section{Effect of nonpredictive cues on target activity in the SC}

Although there is a clear effect of nonpredictive cues on the absolute peak of the target-aligned burst of activity shortly after target onset (Fig. 7), what is still uncertain is its effect on the actual sensory response to the target, independent of pretarget activation. An increase in pretarget activation that often follows the visual-cue response raises the baseline for incoming sensory signals related to target presentation, potentially 

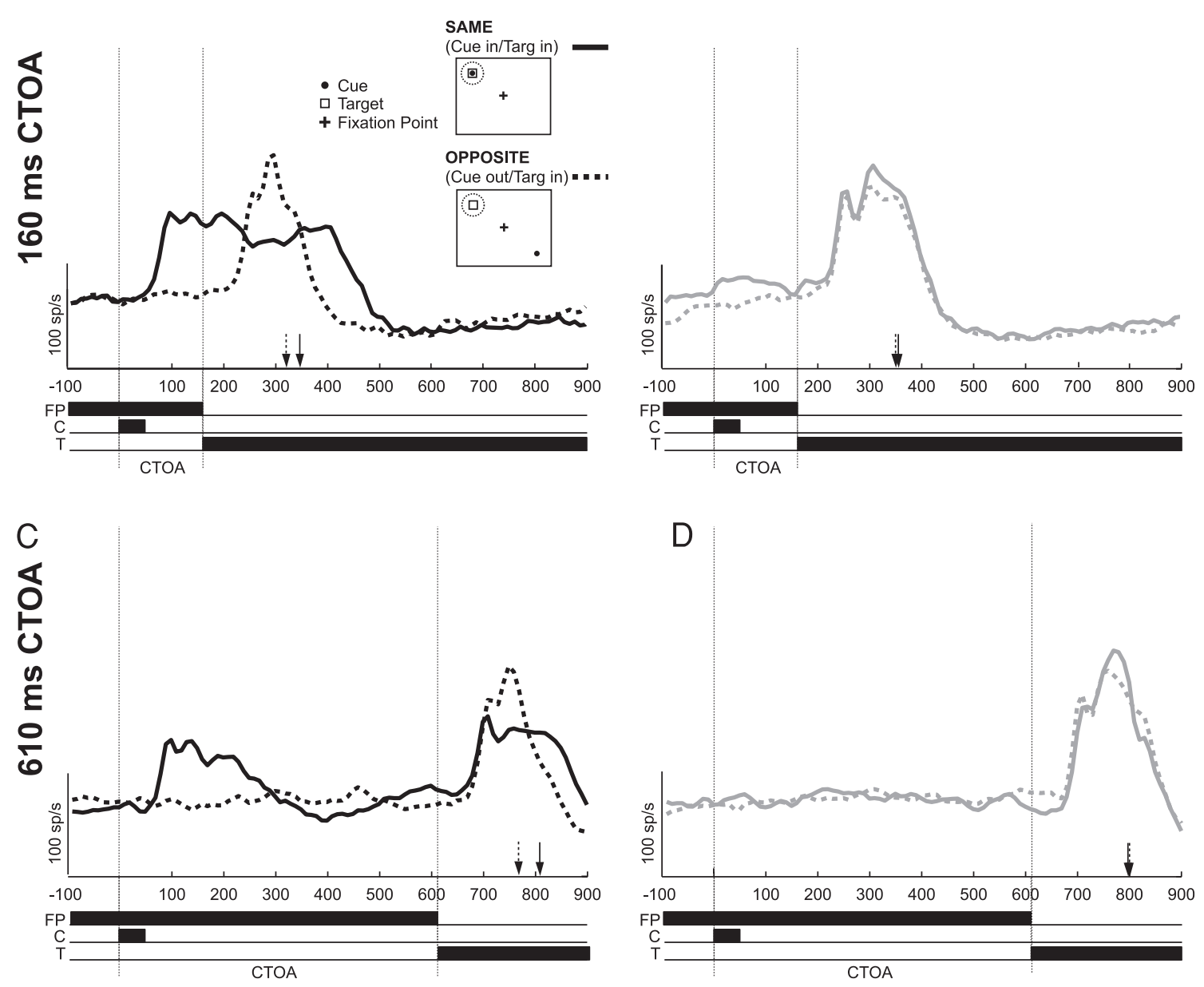

Time from Cue Onset (ms)

FIG. 5. Neuronal correlates of inhibition of return (IOR) in the SC. Activity for an individual sensory-motor neuron recorded from the intermediate layers of the SC, for trials at the 160- and 610-ms CTOA. All traces are aligned on cue onset. When a visual cue and target appear in the same receptive field at a CTOA of $160 \mathrm{~ms}$ ( $A$, solid black line), there is a substantial reduction in the magnitude of the target-aligned activity compared with when the cue appeared in the oPPosite location ( $A$, dashed black line). When the CTOA was increased to $610 \mathrm{~ms}$, the difference in magnitude of the target-aligned burst of activity across the two conditions was reduced compared with the previous CTOA. No such changes in activity were observed after auditory cues $(B, D$, gray lines). Vertical arrows indicate the mean SRT for the SAME (solid arrow) and OPPOSITE (dashed arrow) conditions.

masking any changes in the sensory response to the target when it appears at the SAME location (e.g., at the 610-ms CTOA; Fig. 6A). To investigate the effect of nonpredictive cues on the magnitude of the sensory response to the target, we subtracted the pretarget activity (see METHODS) from the peak of the target-aligned burst to isolate the sensory response to the target. This procedure has been schematized in Fig. 9A. The thick, dark arrow represents the epoch where the pretarget activity is measured. The wider, lighter-shaded box represents the epoch where the peak of the target-aligned burst of activity is measured. Subtracting the former from the latter reveals the magnitude of the sensory response to the target (solid and dashed vertical arrows for the SAME and OPPOSITE conditions, respectively).

This analysis revealed a knockdown of the target-related response across all CTOAs when the visual cue and target appeared at the SAmE location (Fig. 9, $B$ and $C$ ). Although this effect was statistically significant at all CTOAs, it was strongest at the 160-ms CTOA. Interestingly, the difference at the 160-ms CTOA was attributed both to a significant decrease in the response magnitude in the SAME condition (solid black line) and an increase in the opPosite condition (dashed black line) for the corresponding trial type at the 60-ms CTOA (Wilcoxon signed rank-sum test, $P<0.005$ and $P<0.05$, respectively; Fig. 9B). Furthermore, as was predicted in the previous section, isolating the target-related response revealed a significant knockdown of the sensory response to the target at the 610-ms CTOA that was previously masked by the pretarget activity. In the case of auditory-cue trials, although the target-related response was consistently stronger when the cue was presented to the OPPOSITE location compared with the SAME condition, the difference between the two conditions was not statistically significant at any of the CTOAs $(P$ values $>0.10)$.

To summarize, these results demonstrate that when a visual 


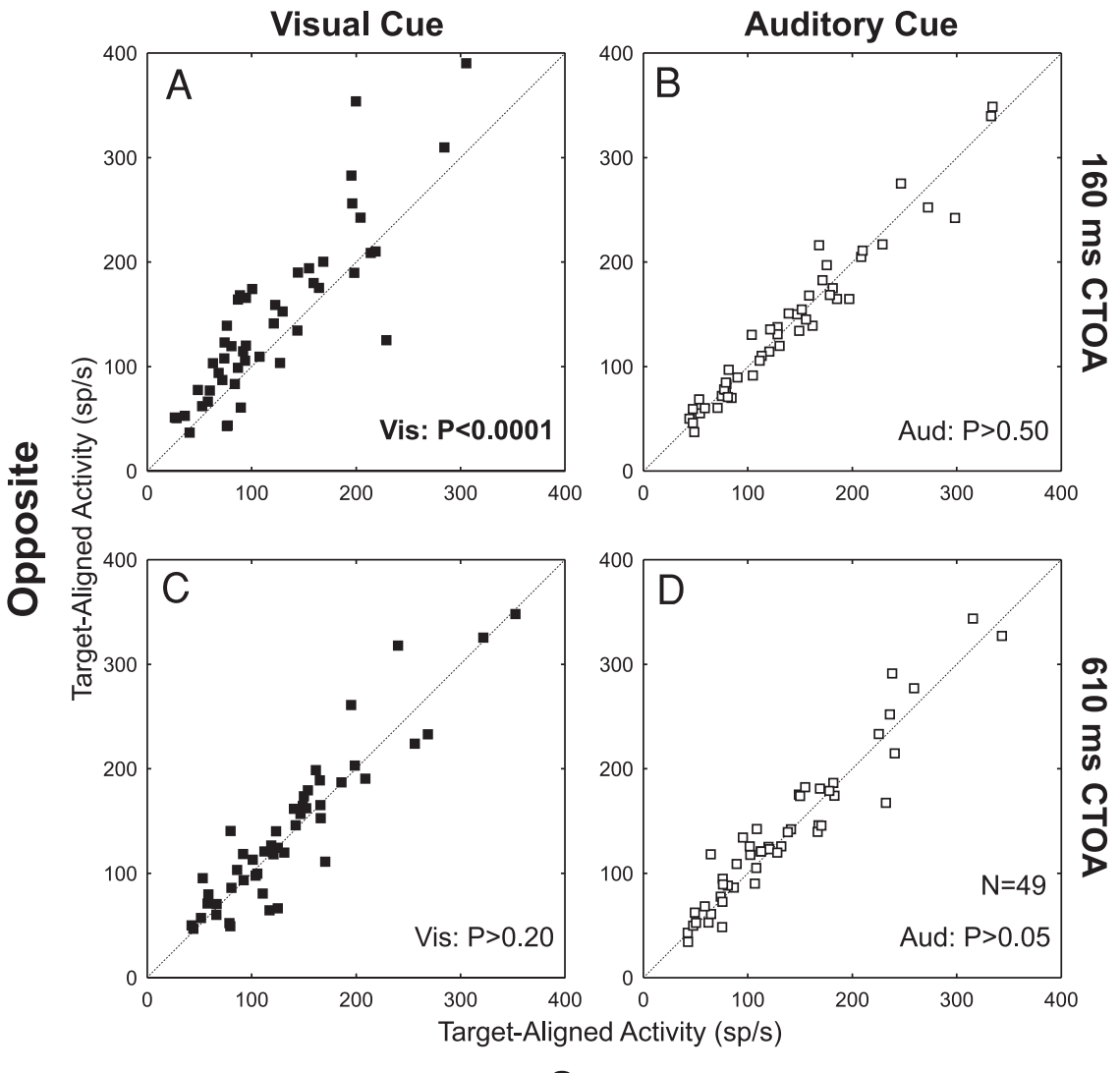

FIG. 6. Influence of cue condition and modality on neuronal activity at the 160- and 610-ms CTOA. Each point represents the mean peak in the target-aligned burst of activity for an individual visually responsive neuron, after a visual $(A, C)$ or auditory $(B, D)$ cue. Points lying below the unity line indicate greater activity in the SAME condition and vice versa. Across the population, there was a highly significant trend for a stronger burst of target-aligned activity in the OPPOSITE condition compared with the SAME condition after visual cues at the 160-ms CTOA ( $A$, solid points, Wilcoxon signed rank-sum test; $P<0.005$ ) that was no longer significant at the 610-ms CTOA $(C, P>0.20)$. No change in activity was observed after auditory cues at either CTOA $(B, D$, open points). target is preceded by a visual cue presented to the SAME location, the sensory response to that target, ignoring the effect of changes in the pretarget activity, is attenuated compared with when the cue is presented to the opposite location. However, when this response builds on elevated levels of pretarget activity, this attenuation is masked, which can partially counteract the IOR effect (Fig. 2). When a visual target is presented to the OPPOSITE location as a visual cue at a CTOA of $160 \mathrm{~ms}$, the target-related response is enhanced compared with the response at the previous CTOA, which can further increase the magnitude of the IOR effect.

\section{I S C U S S I O N}

The goal of this study was to assess how interactions between the cue- and target-related responses contribute to the behavioral consequences of the reflexive orienting of attention. Monkeys were trained to perform a nonpredictive cue-target saccade task while single-unit activity was recorded from the intermediate layers of the SC. When presented with visual cues at a 60-ms CTOA, monkeys show a same-location advantage in SRT (Fig. 2), consistent with the behavior previously described in the human literature (Jonides 1981; Posner and Cohen 1984; Wright and Richard 2000). This reflexive capture of attention was associated with a stronger target-aligned burst of activity in the SC originating from the overlap of the cueand target-aligned activities (Figs. 3 and 4). At the 160- and 610-ms CTOAs, monkeys exhibited a same-location disadvantage, consistent with the human literature (Maylor and Hockey 1985; Posner and Cohen 1984; Wright and Richard 2000). This IOR effect was associated with a reduction in the magnitude of the target-aligned burst of activity (Figs. 5 and 6). Auditory cues used in this study did not elicit either behavioral effect, nor were any significant differences in the target-aligned burst observed at any of the CTOAs tested.

On the basis of these observations, we hypothesize that interactions between the cue- and target-related sensory signals produce these changes in behavior. Compared with visual stimuli, auditory stimuli evoke weaker responses that occur earlier in time (Table 1). We propose that these different characteristics do not allow for the necessary interactions between the auditory cue and the visual target responses to take place. We discuss how our data support this hypothesis by first showing how these interactions lead to changes in the magnitude of the target-aligned response followed by a description of how changes in this activity might lead to changes in behavior. We also discuss why the auditory stimuli used in this study fail to elicit attentional capture or IOR.

\section{Attentional capture corresponds with overlap of cue and target responses}

Numerous behavioral studies have demonstrated that the sudden onset of a stimulus reflexively captures our attention (Jonides 1981; Jonides and Yantis 1988; Yantis and Jonides 1984, 1990). The early appearance of this effect and its independence from the intentions of the observer (Remington et al. 1992; Theeuwes 1991, 1992) suggest that the capture of attention is a stimulus-driven, "bottom-up" mechanism (Fecteau and Munoz 2003).

Using the cue-target task, we have shown that the capture of attention is linked to a strong target-aligned signal that origi- 

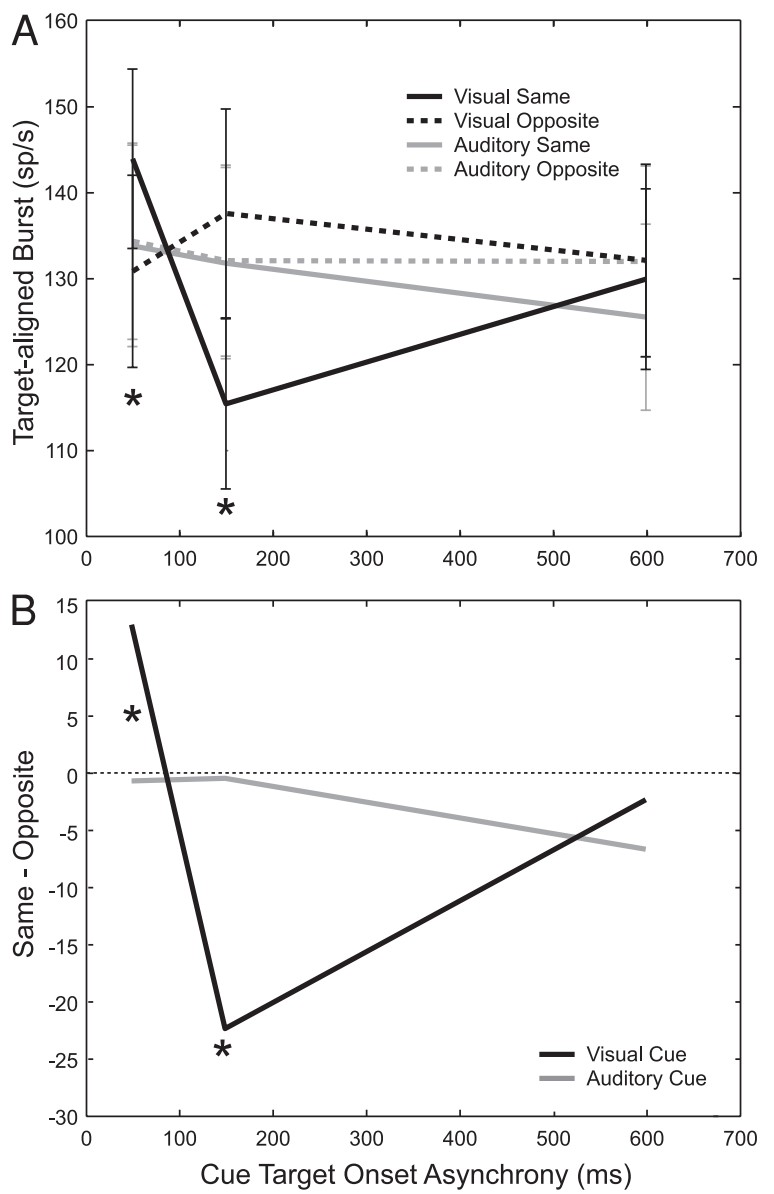

FIG. 7. Population activity in nonpredictive cue-target saccade task. Mean (and SE) peak target-aligned activity for all visually responsive neurons $(A)$. Visual-cue trials are shown in black; auditory-cue trials are shown in gray. Solid lines indicate trials where the cue and target appeared in the SAME location; dashed lines represent trials where the cue and target appeared in OPPOSITE locations (target in receptive field). $B$ : mean activity for the OPPOSITE condition subtracted from that of the SAME condition for peak target-aligned activity. Values $<0$ indicate greater activity in the OPPOSITE condition; values $>0$ indicate greater activity in the SAME condition. Asterisks indicate statistically significant differences between the SAME and OPPOSITE conditions (Wilcoxon rank-sum test, $P<0.05$ ).

nates from the interaction of the cue- and target-aligned activities. This interaction is schematized in Fig. 10. Visual cues elicit a robust sensory response in $\mathrm{SC}$ neurons that occurs about $75 \mathrm{~ms}$ after the appearance of the cue. This response has been shown for the population of visually responsive neurons in Fig. $10 \mathrm{~A}$. Because this initial phasic response to the visual cue persists for $\geq 50-100 \mathrm{~ms}$, cue-related activity will still be present when the target-related response is registered by the neuron after a 60-ms CTOA. This is shown as a solid black line in Fig. 10B. The overlap between the cue- and target-aligned activities allows the incoming target signal to build on the cue-related activity resulting in a significantly stronger targetaligned burst of activity compared with when the cue and target appear at OPPOSITE locations ("Target," Fig. 10B). The magnitude of the target-aligned burst in the OPPOSITE condition (dashed black line, Fig. 10B) is further reduced, presumably attributable to the local inhibitory network in the SC (Munoz and Istvan 1998) that inhibits other areas of the SC when one subpopulation is active (i.e., when the contralateral SC is responding to the cue, the ipsilateral SC will be inhibited), shown as the drop in baseline activity in Fig. 10B. Therefore because the level of activity is reduced, additional time is required to trigger a saccade.

The consequences of auditory cues, or lack thereof, strongly support our contention. Compared with visual stimuli, auditory stimuli elicit weaker responses that occur about $45 \mathrm{~ms}$ after the stimulus appeared (Table 1). Moreover, so few neurons responded to the auditory stimuli resulting in a much weaker response across the population, shown for the population of aurally responsive neurons in Fig. 10A. Therefore there will be no residual activity left for the target response to build on and consequently no modulation of the response after auditory cues; hence no change in behavior is observed (gray lines, Fig. $10 B$ ) and so the capture of attention is not observed in our study.

\section{IOR corresponds to a weaker target-aligned response}

Involvement of the SC in IOR was first proposed by Posner and colleagues (1985) (see also Rafal et al. 1988) on the basis of clinical findings. They found that patients suffering from progressive supranuclear palsy, a Parkinson's-like disorder that affects various brain stem structures including the SC (Burn and Lees 2002), did not exhibit IOR under conditions when normal subjects or patients with Parkinson's disease did. Since then, converging lines of evidence from both clinical and neurophysiological studies suggest a role for the SC in IOR (Dorris et al. 2002; Sapir et al. 1999). However, although we
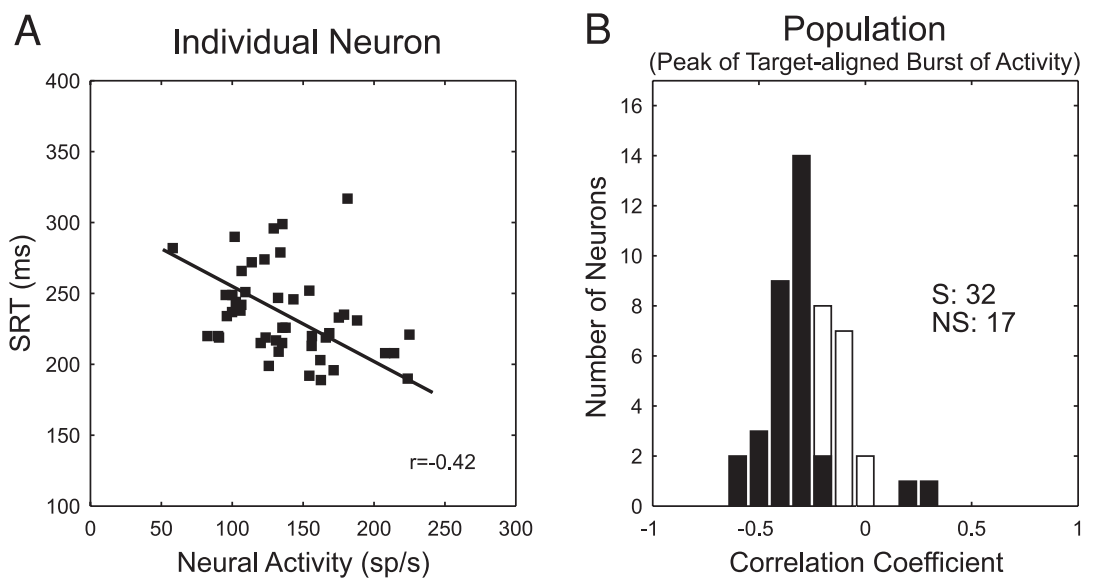

FIG. 8. Correlation between saccadic reaction time and neural activity in the SC. A: trial-by-trial correlation of SRT vs. peak magnitude of the target-aligned burst of activity for a single sensory-motor neuron recorded from the intermediate layers of the SC at the 160-ms CTOA. This neuron displayed a significant negative correlation between SRT and neural activity. $B$ : histogram of the Pearson correlation coefficients for SRT vs. target-aligned burst of activity for the population of visually responsive neurons. Negative values indicate that shorter SRTs are correlated with greater activity. Individual neurons with significant correlations $(P<$ 0.05 ) are represented as solid bars. 
observe a neural correlate of IOR in the SC, what specific role does the SC play?

When a visual target appears at the SAME location as the
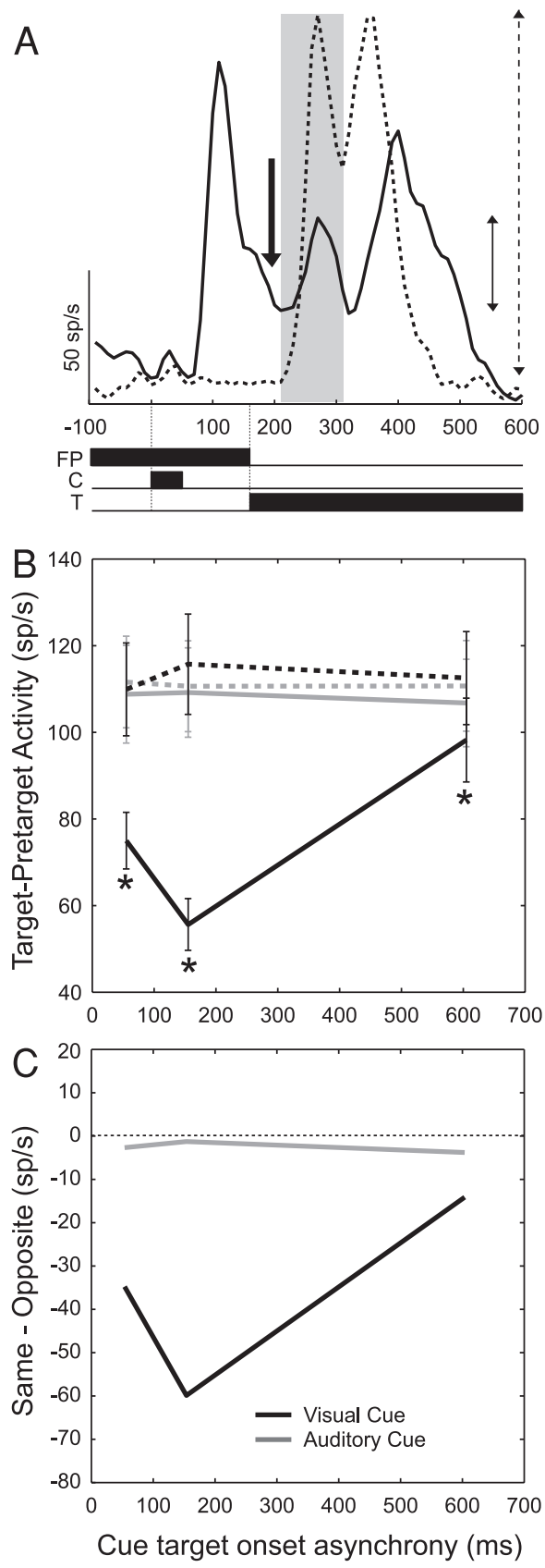

FIG. 9. Effect of nonpredictive cues on the sensory response to the target. $A$ : activity of a sensory-motor neuron showing how pretarget activation after visual-cue onset elevates the baseline for the target response in the SAME condition. Solid and dashed vertical arrows indicate the target-related response (i.e., equal to the pretarget activity subtracted from the peak target-aligned burst of activity) for the SAME and OPPOSITE conditions, respectively. $B$ : mean (and SE) target-related response magnitude for all visually responsive neurons. Visual-cue trials are shown in blackl auditory-cue trials are shown in gray. Solid lines indicate trials where the cue and target appeared in the SAME locationl dashed lines represent trials where the cue and target appeared in OPPOSITE locations. $C$ : mean activity for the OPPOSITE condition subtracted from that of the SAME condition for peak target-aligned activity. Values $<0$ indicate greater activity in the OPPOSITE condition; values $>0$ indicate greater activity in the SAME condition. Asterisks indicate statistically significant differences between the SAME and OPPOSITE conditions (Wilcoxon rank-sum test, $P<0.05$ ).

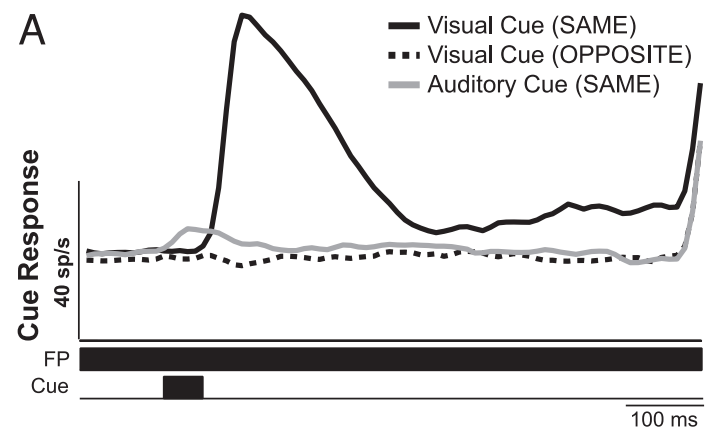

B Attentional Capture

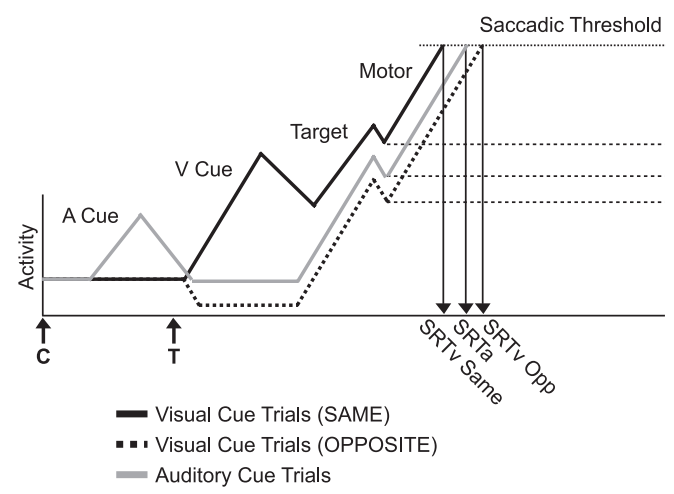

C

Inhibition of Return

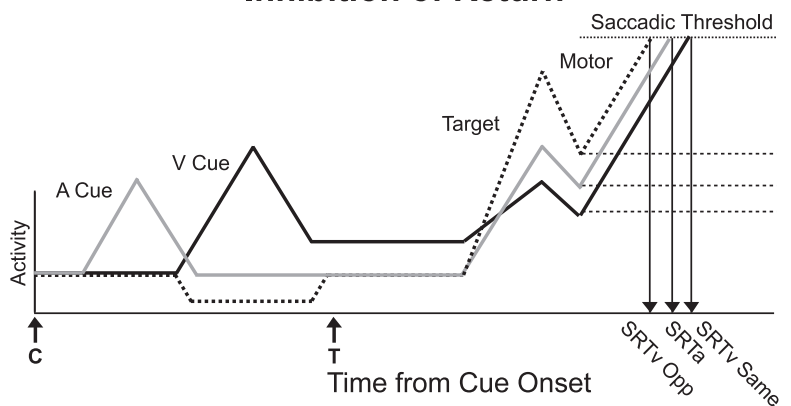

FIG. 10. Proposed mechanism for attentional capture and inhibition of return in the SC. A: visual and auditory cue-aligned responses for the population of visual and bimodal neurons $(n=49)$. Visual cues evoke a robust, phasic sensory response that is often followed by increasing pretarget activity. Auditory cues evoke short latency, weak, and abrupt responses. B: proposed mechanism for attentional capture. When a visual cue (solid black line) is presented into the response field of neurons in the SC, they respond with a burst of action potentials. This suppresses the activity of neurons that are not responding to the cue (shown as the drop in baseline for the OPPOSITE condition, dashed black line). When the target later appears, summation of the cue- and target-aligned responses increases the level of activity so that when the motor-related activity begins to accumulate; a saccade can be triggered sooner compared with the other conditions. No such overlap of the cue- and target-aligned responses occurs after auditory cues; hence no attentional capture is seen. Likewise, because of the suppression in the oPPOSITE condition, the level of activity is reduced and additional time is required to trigger a saccade. $C$ : inhibition of return in response to visual cues occurs because of the reduction in the target-aligned response, which, despite the presence of residual cue activity, still results in significantly reduced activity. This reduces the level of activity on which the motor-related activity will build and consequently increases the time required to surpass saccadic threshold and trigger a saccade. There is no modulation of the response after auditory cues; hence no change in behavior is observed.

visual cue, the magnitude of the target-aligned burst was significantly reduced compared with when the cue and target appear at OPPOSITE locations (Fig. 7; see also Dorris et al. 2002; 
Fecteau and Munoz 2003). This reduction occurred despite an increase in pretarget activity (Fig. 9) so that the net activity was still weaker compared with when the two stimuli are presented to OPPOSITE locations, particularly at the 160-ms CTOA. A weaker target-aligned burst of activity provides a lower amount of activity on which the motor-related activity will build and as such, the time required to trigger a saccade is increased (Fig. $10 C$, see following text). This reduction in activity was not observed after auditory cues, which accounts for why the SRTs for auditory-cue trials were unaffected. Furthermore, it appeared that the OPPOSITE condition was facilitated (see following text), which further augmented the IOR effect. These data represent a link between a sensory event and the ultimate behavior, supporting the theory that IOR in this particular oculomotor task is driven by sensory interactions between the cue and target.

One question that has yet to be addressed is what is/are the physiological mechanism(s) underlying this modulation of the target-related response after a visual but not auditory cue? One immediately obvious possibility is that visuomotor neurons in the SC are directly inhibited, either from local sources (e.g., fixation neurons in the rostral SC; Munoz and Wurtz 1993) or by external projections (e.g., substantia nigra pars reticulata; Hikosaka and Wurtz 1983; Wurtz and Hikosaka 1986). However, Dorris and colleagues (2002) provided critical evidence suggesting this is not the case. In a similar cue-target saccade task as used in the current study, they applied microstimulation to the SC instead of presenting a target for the purposes of evoking a saccade. Contrary to what might be expected if the $\mathrm{SC}$ were being directly inhibited, they found that evoked latencies were shorter when stimulation was applied to the SAME side as that which responded to the cue, which is likely attributable to the microstimulation combining with increases in pretarget activity.

Another possibility therefore is the $\mathrm{SC}$ is receiving reduced visual inputs from structures earlier in the visual pathway (e.g., retina, lateral geniculate nucleus, primary visual cortex). This is supported by previous findings showing similar reductions in the target responses of neurons in the superficial layers of the SC (Dorris et al. 2002; Robinson and Kertzman 1995) and parietal cortex (Robinson et al. 1995) and could therefore represent a common feature of the magnocellular pathway. Neurons in this pathway may be unable to respond fully to the target when presented to the SAME location. Because this pathway is less concerned with physical properties of visual stimuli (e.g., color, shape) and more with spatial and temporal relationships between stimuli, this sensory-based mechanism may help account for the observations that led researchers to theorize that IOR is an adaptive strategy meant to maximize the efficiency of visual search (see Klein 2000). The absence of such an effect after auditory cues further supports a reduction in visual inputs driving the knockdown of the visual response in IOR. Neurons in the intermediate layers of the SC represent one of the earliest sites of crossmodal convergence at the level of the individual neuron. Sensory neurons earlier in the visual and auditory pathways will respond to visual or auditory stimuli-but not both - and so the auditory cue should have no direct effect on the discharge properties of neurons earlier in the visual pathway.

\section{How do changes in the target-aligned responses link to changes in SRT?}

In the preceding sections, we have described how interactions between the cue and target result in changes to the magnitude of the target-aligned burst. How then do these changes result in changes in behavior? Previous studies have suggested that neural activity must accumulate toward a certain level of activity, defined as the saccadic threshold, before a saccade will be initiated (Carpenter and Williams 1995; Gold and Shadlen 2000; Hanes and Schall 1996). Factors that affect when saccadic threshold is exceeded will therefore have a strong influence on SRT.

Shortly after the onset of the target-aligned burst, activity related to the generation of the motor output will begin to accumulate toward saccadic threshold ("Motor"; Fig. 10, $B$ and $C$ ). Even though the target-aligned burst may have decayed somewhat by this time, the "starting point" (horizontal dashed lines, Fig. 10, $B$ and $C$ ) for the motor activity will still be linked to the magnitude of the initial target-aligned burst. When the target-aligned response is strong, as is the case with the SAME condition at the 60-ms CTOA (Fig. 10B) and with the OPPOSITE condition at the 160- and 610-ms CTOAs (Fig. 10C), the neural activity will exceed saccadic threshold first, thus triggering a saccade with a shorter SRT.

\section{Facilitation to novel locations?}

Another facet of IOR that we have not considered up to now is the facilitated responding to the OPPOSITE side (i.e., novel locations; see Bennett and Pratt 2001; Pratt and Abrams 1999). At the 160-ms CTOA, where IOR was strongest, in addition to observing a reduction in the magnitude of the target-aligned activity in the SAME condition that was associated with an increase in SRT, we also observed an increase in the magnitude of the target-aligned activity (Fig. 7) coupled with a decrease in SRT for the opPosite condition (Fig. 2) compared with the auditory-cue trials or the visual-cue trials at the other CTOAs. One possibility is that this facilitation is being generated locally, within the SC. While one subpopulation of neurons in the $\mathrm{SC}$ are responding to the cue, neurons in the opposite side SC are being inhibited because of the widespread inhibitory network (Mize et al. 1991; Munoz and Istvan 1998). When the visual target later appears at the opPosite location as the cue, neurons that were previously inhibited may be able to respond with greater activity because of a postinhibitory rebound/excitation mechanism (e.g., Nishimura et al. 1992; Okada et al. 1990; Syed et al. 1990). Biophysical studies have demonstrated that, after a period of inhibition, neurons can discharge with increased frequency compared with whether no previous inhibition was present. For example, in a study examining saccadic suppression, the phenomenon whereby visual inputs are suppressed during saccadic eye movements, Zhu and Lo (1996) demonstrated that stimulating the deeper layers of the SC resulted in inhibition of neurons in the lateral geniculate nucleus that was followed by a period of facilitation shortly after the initial stimulation pulse. If such a mechanism were functioning in our task, this could be expressed as an enhanced target-related response over that obtained in the neurons that responded to the cue. Further investigation will be necessary to support or refute this theory but it nonetheless illustrates how 
sensory processes related to the cue response might result in changes in the magnitude of the target-aligned burst of activity and subsequent changes in behavior.

\section{Why no crossmodal attentional biases in our task?}

The monkeys in our study did not exhibit either reflexive attentional capture or IOR in response to auditory cues. Early crossmodal facilitation has been demonstrated in detection tasks (e.g., Macaluso et al. 2000; McDonald et al. 2000). However, in the current study that used an oculomotor task, the auditory cues used failed to evoke reflexive attentional capture, presumably because the amount of residual activity in the SC after the auditory cue was insufficient to significantly affect the magnitude of target-aligned activity at the $60-\mathrm{ms}$ CTOA, which would have directly affected the motor output of the SC. The lack of IOR after auditory cues can also be accounted for by the differences in the visual versus auditory-cue response properties and their subsequent effect on the target-related response, as outlined in the previous sections.

This does not, however, explain why several other studies using humans have been able to elicit crossmodal IOR after presentation of an auditory cue (e.g., Reuter-Lorenz et al. 1996; Spence and Driver 1998; Tassinari et al. 2002). The specific task conditions in these aforementioned studies differ significantly from our own and it is likely that these differences may account for their ability, and our failure, to evoke crossmodal IOR. For example, using a visual detection task, Reuter-Lorenz and Rosenquist (1996) were unable to induce IOR after auditory cues unless subjects were instructed to first make saccades to the cue and then return to fixation before target presentation. They argued that auditory stimuli alone were unable to sufficiently activate the requisite oculomotor systems necessary to induce IOR. However, by having subjects generate an oculomotor response to the cue, the oculomotor system was engaged and therefore IOR was presumably more likely to occur.

Similarly, Spence and Driver (1998) demonstrated IOR to a visual target after an auditory cue if an auditory fixation point was used to redirect attention back to fixation after cue presentation. IOR was not observed, however, if a visual fixation point was used to redirect attention. In this case, the auditory modality is given increased salience, not because it was the target for a saccade but because it was being used to draw attention to several different locations in a given trial and was critical to the subjects' being able to perform the task correctly. It should be noted that in both above cases, subjects were required to generate responses to auditory stimuli, whether they were overt orienting movements or detection responses. We would argue that this fact alone introduces additional influences that will affect the outcome.

In conclusion, altogether we have shown that the summation of overlapping sensory signals in the SC can result in attentional capture. We have confirmed that IOR corresponds to reductions in the target-aligned response that scale with the difference in SRT. Finally, we have provided a neurophysiological basis for why the auditory stimuli used in our study fail to evoke either effect. Although these results support the involvement of the SC in reflexive covert orienting, it remains to be seen what is driving the changes in the target-related responses.

\section{A C K N OW LED GMENTS}

We thank A. Lablans, R. Pengally, and F. Paquin for invaluable assistance and technical expertise; and I. T. Armstrong, S. Boehnke, B. Coe, J. Gore, R. Marino, and K. Rodgers for commenting on earlier versions of this manuscript.

\section{G R A N T S}

This work was supported by a Human Frontiers Science Program Grant RG0174/1998-B. A. H. Bell was supported by a Doctoral Research Award from the Canadian Institutes of Health Research. J. H. Fecteau was supported by a Postdoctoral Fellowship from the Natural Sciences and Engineering Research Council of Canada.

\section{REFER EN C ES}

Bahill A, Clark M, and Stark L. The main sequence, a tool for studying human eye movements. Math Biosci 24: 191-204, 1975.

Bell AH, Corneil BD, Meredith MA, and Munoz DP. The influence of stimulus properties on multisensory processing in the awake primate superior colliculus. Can J Exp Psychol 55: 123-132, 2001.

Bell AH, Corneil BD, Munoz DP, and Meredith MA. Engagement of visual fixation suppresses sensory responses and multisensory integration in the primate superior colliculus. Eur J Neurosci 18: 2867-2873, 2003.

Bell AH, Meredith MA, Van Opstal AJ, and Munoz DP. Role of the primate superior colliculus in mediating multimodal orienting behaviour. Neural Control Move Abstr 2002.

Bell AH and Munoz DP. Activity in the primate superior colliculus related to performance in predictive vs. non-predictive multimodal cued-saccade tasks. Soc Neurosci Abstr 27: 560.5, 2002.

Bennett PJ and Pratt J. The spatial distribution of inhibition of return. Psychol Sci 12: 76-80, 2001.

Burn DJ and Lees AJ. Progressive supranuclear palsy: where are we now? Lancet Neurol 1: 359-369, 2002.

Carpenter RH and Williams ML. Neural computation of log likelihood in control of saccadic eye movements. Nature 377: 59-62, 1995.

Crist CF, Yamasaki DS, Komatsu H, and Wurtz RH. A grid system and a microsyringe for single cell recording. J Neurosci Methods 26: 117-122, 1988.

Dorris MC, Klein RM, Everling S, and Munoz DP. Contribution of the primate superior colliculus to inhibition of return. J Cogn Neurosci 14: 1256-1263, 2002.

Egeth HE and Yantis S. Visual attention: control, representation, and time course. Annu Rev Psychol 48: 269-297, 1997.

Fecteau JH and Munoz DP. Neurophysiological correlates of covert orienting to uninformative and informative cues in the primate superior colliculus. Cogn Neurosci Annu Meet 10: 2003.

Gold JI and Shadlen MN. Representation of a perceptual decision in developing oculomotor commands. Nature 404: 390-394, 2000.

Hanes DP and Schall JD. Neural control of voluntary movement initiation [see comments]. Science 274: 427-430, 1996.

Hays AV, Richmond BJ, and Optican LM. A UNIX-based multiple process system for real-time data acquisition and control. WESCON Conf Proc 2: $1-10,1982$.

Hikosaka $\mathrm{O}$ and Wurtz RH. Visual and oculomotor functions of monkey substantia nigra pars reticulata. IV. Relation of substantia nigra to superior colliculus. J Neurophysiol 49: 1285-1301, 1983.

Jonides J. Voluntary versus automatic control over the mind's eye's movement. In: Attention and Performance, vol. IX, edited by Long JB and Baddeley AD. Hillsdale, NJ: Erlbaum Associates, 1981, p. 187-203.

Jonides $\mathbf{J}$ and Yantis S. Uniqueness of abrupt visual onset in capturing attention. Percept Psychophys 43: 346-354, 1988.

Klein RM. Inhibition of return. Trends Cogn Sci 4: 138-147, 2000.

Macaluso E, Frith CD, and Driver J. Modulation of human visual cortex by crossmodal spatial attention. Science 289: 1206-1208, 2000.

Maylor EA and Hockey R. Inhibitory component of externally controlled covert orienting in visual space. J Exp Psychol Hum Percept Perform 11: 777-787, 1985.

McDonald JJ, Teder-Salejarvi WA, and Hillyard SA. Involuntary orienting to sound improves visual perception. Nature 407: 906-908, 2000.

Mize RR, Jeon CJ, Hamada OL, and Spencer RF. Organization of neurons labeled by antibodies to gamma-aminobutyric acid (GABA) in the superior colliculus of the Rhesus monkey. Vis Neurosci 6: 75-92, 1991.

Munoz DP and Istvan PJ. Lateral inhibitory interactions in the intermediate layers of the monkey superior colliculus. J Neurophysiol 79: 1193-1209, 1998. 
Munoz DP and Wurtz RH. Fixation cells in monkey superior colliculus. I. Characteristics of cell discharge. J Neurophysiol 70: 559-575, 1993.

Nishimura S, Okada Y, and Amatsu M. Post-inhibitory excitation of adenosine on neurotransmission in guinea pig hippocampal slices. Neurosci Lett 139: $126-129,1992$.

Okada Y, Nishimura S, and Miyamoto T. Excitatory effect of adenosine on neurotransmission in the slices of superior colliculus and hippocampus of guinea pig. Neurosci Lett 120: 205-208, 1990.

Poliakoff E, Spence C, O'Boyle DJ, McGlone FP, and Cody FW. Tactile inhibition of return: non-ocular response inhibition and mode of response. Exp Brain Res 146: 54-59, 2002.

Posner MI. Chronometric Explorations of Mind: The Third Paul M. Fitts Lectures, delivered at the University of Michigan, Ann Arbor, MI, September 1976. Hillsdale, NJ: Erlbaum Associates, 1978.

Posner MI and Cohen Y. Components of visual orienting. In: Attention and Performance, vol. X, edited by Bouma $\mathrm{H}$ and Bouwhuis DG. Hillsdale, NJ: Erlbaum Associates, 1984, p. 531-556.

Posner MI, Rafal RD, Choate LS, and Vaughan J. Inhibition of return: neural basis and function. Cogn Neuropsychol 2: 221-228, 1985.

Pratt J and Abrams RA. Inhibition of return in discrimination tasks. $J$ Exp Psychol Hum Percept Perform 25: 229-242, 1999.

Rafal RD, Posner MI, Friedman JH, Inhoff AW, and Bernstein E. Orienting of visual attention in progressive supranuclear palsy. Brain 111: 267-280, 1988.

Remington RW, Johnston JC, and Yantis S. Involuntary attentional capture by abrupt onsets. Percept Psychophys 51: 279-290, 1992.

Reuter-Lorenz PA, Jha AP, and Rosenquist JN. What is inhibited in inhibition of return? J Exp Psychol Hum Percept Perform 22: 367-378, 1996.

Reuter-Lorenz PA and Rosenquist JN. Auditory cues and inhibition of return: the importance of oculomotor activation. Exp Brain Res 112: 119126, 1996.

Robinson DL, Bowman EM, and Kertzman C. Covert orienting of attention in macaques. II. Contributions of parietal cortex. J Neurophysiol 74: 698 712, 1995.

Robinson DL and Kertzman C. Covert orienting of attention in macaques. III. Contributions of the superior colliculus. J Neurophysiol 74: 713-721, 1995.

Sapir A, Soroker N, Berger A, and Henik A. Inhibition of return in spatial attention: direct evidence for collicular generation. Nat Neurosci 2: 1053 1054, 1999.
Spence $\mathbf{C}$ and Driver J. Inhibition of return following an auditory cue. The role of central reorienting events. Exp Brain Res 118: 352-360, 1998.

Spence C, Lloyd D, McGlone F, Nicholls ME, and Driver J. Inhibition of return is supramodal: a demonstration between all possible pairings of vision, touch, and audition. Exp Brain Res 134: 42-48, 2000.

Spence C, Nicholls ME, Gillespie N, and Driver J. Cross-modal links in exogenous covert spatial orienting between touch, audition, and vision. Percept Psychophys 60: 544-557, 1998.

Stein BE and Meredith MA. The Merging of the Senses. Cambridge, MA: MIT Press, 1993.

Syed NI, Bulloch AG, and Lukowiak K. In vitro reconstruction of the respiratory central pattern generator of the mollusk Lymnaea. Science 250: 282-285, 1990.

Tassinari G, Campara D, Benedetti C, and Berlucchi G. The contribution of general and specific motor inhibitory sets to the so-called auditory inhibition of return. Exp Brain Res 146: 523-530, 2002.

Theeuwes J. Cross-dimensional perceptual selectivity. Percept Psychophys 50: 184-193, 1991.

Theeuwes J. Perceptual selectivity for color and form. Percept Psychophys 51: 599-606, 1992.

Thompson KG, Hanes DP, Bichot NP, and Schall JD. Perceptual and motor processing stages identified in the activity of macaque frontal eye field neurons during visual search. J Neurophysiol 76: 4040-4055, 1996.

Waitzman DM, Ma TP, Optican LM, and Wurtz RH. Superior colliculus neurons mediate the dynamic characteristics of saccades. J Neurophysiol 66: 1716-1737, 1991.

Wallace MT, Wilkinson LK, and Stein BE. Representation and integration of multiple sensory inputs in primate superior colliculus. J Neurophysiol 76: 1246-1266, 1996.

Wright RD and Richard CM. Location cue validity affects inhibition of return of visual processing. Vision Res 40: 2351-2358, 2000.

Wurtz RH and Hikosaka O. Role of the basal ganglia in the initiation of saccadic eye movements. Prog Brain Res 64: 175-190, 1986.

Yantis $\mathbf{S}$ and Jonides J. Abrupt visual onsets and selective attention: evidence from visual search. J Exp Psychol Hum Percept Perform 10: 601-621, 1984.

Yantis $\mathbf{S}$ and Jonides J. Abrupt visual onsets and selective attention: voluntary versus automatic allocation. J Exp Psychol Hum Percept Perform 16: 121-134, 1990. 